\title{
An Improved Modelling of User Clustering For Small Cell Deployment In Heterogeneous Cellular Network.
}

Joyatri Bora ( $\sim$ bjoyatri@yahoo.com )

North Eastern Regional Institute of Science and Technology https://orcid.org/0000-0001-6881-8595

Anwar Hussain

North Eastern Regional Institute of Science and Technology

\section{Research Article}

Keywords: Heterogeneous, Clustering, K-means Algorithm, Doubly Stochastic Poison Process, Shadow Fading

Posted Date: June 3rd, 2021

DOI: https://doi.org/10.21203/rs.3.rs-476123/v1

License: (c) (i) This work is licensed under a Creative Commons Attribution 4.0 International License.

Read Full License 


\section{An Improved Modelling Of User Clustering for Small Cell Deployment In Heterogeneous Cellular Network}

\author{
Joyatri Bora \\ Electronics and Communication Engg. Dept. \\ North Eastern Regional Institute of Science and \\ Technology \\ Nirjuli-791109, Arunachal Pradesh, India \\ bjoyatri@yahoo.com
}

\author{
Md. Anwar Hussain \\ Electronics and Communication Engg. Dept. \\ North Eastern Regional Institute of Science and \\ Technology \\ Nirjuli-791109, Arunachal Pradesh, India \\ bubuli_99@yahoo.com
}

Abstract: Users in practical cellular geographical areas are found to be non-uniformly distributed. Small cell $(S C)$ deployments in heterogeneous user distribution in a cellular geographical area help to meet high data rate user demands for multimedia data communications in hot spots. SCs help to offload traffic burden from the macro cell $(M C)$ base station, and also cater the data traffic need for the edge users where signal strength from the $M C$ base station $(B S)$ is very weak. For deployments of $S C s$ along with the central MC BS (hence called HetNet) in such spatial heterogeneous user distribution, effective user grouping or clustering algorithm is required for appropriate and satisfactory service coverage. We call it service grouping or clustering of users to be put under a $S C$ for data transmission and reception. It does not disturb the spatial positions of users in clustered non-uniform distribution. Efficient grouping or clustering of users and then deploying a $S C$ at optimal location enhances the performance of the HetNet. It is found that the K-means algorithm used for such grouping of users to position $S C S$ is not efficient. A novel and improved user grouping algorithm is proposed in this paper which performs much better compared to the $k$-means algorithm. The proposed algorithm of modelling of user clustering results in increase in the number of users under SCs, increase in more offloading of data traffic from $M C B S$ thereby increasing data throughput of $M C$ users. The algorithm also increases in the energy efficiencies of the $S C s$ which is considered as one important performance metric. A doubly stochastic poison process (DSPP) also called Cox process is assumed here for simulation of non-uniform user distributions. We consider Rayleigh distributed small scale fading model, large scale fading factor representing shadow fading, and users' geographical distances from $B S s$ to evaluate users' data rates.

Keywords - Heterogeneous; Clustering; K-means Algorithm; Doubly Stochastic Poison Process; Shadow Fading;

\section{INTRODUCTION}

Presently, cellular mobile data communication has far grown than that expected in the last decade of the twenty first century [1]. The extensive growth of data volume generated due to ever increasing number of smart phone users has inspired the use of larger spectrum and more penetration of sophisticated mobile data communication systems in rural areas in addition to the urban usages. Also, the user distribution in a given geographical area is now more non-uniformly distributed than before, with number of hotspots of users where much activity is recorded and data volume generated significantly higher. Also, in areas near the edge of the geographical area, where signal strength from the center $B S$ is very weak, cellular service coverage is needed [2-3]. Instead of a single $B S$ at the center for coverage of all the users, SCs are also deployed at certain points like the centers of the hotspots or at the cell edge for enhanced throughput or data rate performance of the total cellular data communication system [4-7]. SCs not just 
offload the traffic from a $M C B S$ in the cell but also boost link quality by reducing the gap of communicating distance, and permit to utilize the spectrum in an efficient manner [6-7]. In [8], a novel HetNet planning model is proposed that involves deployment of $M C$ and SCs considering least energy consumption and minimum total cost ownership (TCO) that fulfil the traffic demand. While deploying SCs, factors that are given importance are power and bandwidth allocation, traffic requirement, and spectral efficiency. Three heuristic procedures are adopted along with clustering approaches in [9] for joint deployment of SCs and point-to-point fibre links that meets the minimum level of $Q o S$ requirement as well as reducing cost of transport and SCs deployment. An observerbased system is used dividing a cell into a series of sectors and identifying the regions of mobile users' clusters for finding out the optimum location of deployment of SCs [10]. In order to cope with dense deployment scenario for heavy traffic requirements, $S C s$ within a $M C$ are clustered with grouping of 1, 4 or 10 based on 3GPP simulation methodology [11-12]. Hierarchical clustering algorithm with minimax linkage [13] is used to cluster BSs creating virtual cells based on best channel condition and user affiliation to a BS [14]. A Green Offloading scheme is proposed for offloading traffic from MCs based on Reverse Auction Model [15]. The first price sealed bid mechanism which matches with mobility based offloading process [16] is found to improve system energy efficiency of densely deployed HetNet under the constraints of user $Q o S$ requirements, bandwidth and transmission power limitations. Nowadays, a promising technology of $5 \mathrm{G}$ is hyper-dense SC deployed HetNet. However, with increased number of large $S C B S s$ the level of power consumption also increases. Therefore, $S C B S s$ are operated according to traffic load variations. During the day when user density is high $S C$ $B S s$ are activated gradually to serve offloaded traffic of $M C$ $B S$ and operated in off mode when traffic is low thus reducing total power consumption of the HetNet [17].

Stochastic Modelling of the user distribution and deployments of $S C s$ for network performance evaluation are now prevalent.
Cox process or cluster model is used to model the non-uniform distribution in a cellular area [18-23]. To divide the cellular area in a number of clusters, $k$-means clustering technique with Voronoi cells may be used [6]. SC BSs may now be placed at the centroids of the cells, and the $B S$ caters the data service required by the users with a specific circular area of the SCs. Similarly, SC BSs may be located judiciously near the edge of the $M C$. However, the $k$-means clustering technique has certain defects such as within the circular area of some $S C$ $B S s$, there may be no user at all or the numbers of users are very small. The authors in [24] discuss the impact of SCs on the throughput enhancement in HetNets and have shown the limitation of k-means user clustering approach. It is obvious that the SC BSS should be located in such locations where the users' density is maximum within its $S C$ area. This results in offloading of data traffic from the $M C B S$ maximum possible which is a requirement in cellular communication design of HetNets. Also, by bringing more and more users to the coverage of SCs enhances the data throughput of those users [25-26].

In this paper, we assume a HetNet with a $M C B S$ serving the macro users and a few SCs deployed in the macro geographical area serving users in cluster locations. SCs are placed at the centroid of the clusters of users obtained applying $k$-means algorithm and the proposed algorithm obtained by improving upon the $k$-means algorithm. We obtain the total data throughputs of users under the $M C B S$ and under all the SCs separately, for both the algorithms. The proposed algorithm increases data throughput of macro users, and also increases total number of users under the SCs, which are considered as metrics to show the efficiency of the proposed algorithm. The effective throughput offloading, OFFLOAD and energy efficiency of $S C B S s, B S_{-} S C_{-} W$ are two other metrics used for performance evaluation. We consider different cluster scenarios for evaluation of the proposed algorithm. The user spatial heterogeneity is simulated using Cox process and the $M C B S$ is assumed to be in the center of a circular geographical area. 
The rest of the paper is organized as follows: We describe in Section 2 the system model and modelling of users resulting in the improved user grouping and obtaining optimum location to deploy SCs. In Section 3, the metrics are formulated for evaluation of the new improved modelling of user clustering. Section 4 describes the simulation studies and discussions. Section 5 concludes the findings of this paper.

\section{SYSTEM MODEL}

We consider a circular geographical area of radius 1500 meters which is filled by a non-uniform Cox process that generates users as clusters spatially. A $M C B S$ is positioned at the center and a few SCs of radius 100 meters are located in the clusters of users of the geographical area. The users are taken in different numbers in clusters, the Centroid or Centre of which is obtained with k-means clustering algorithm. An example HetNet is shown in Fig. 1, wherein the red outer circular area is the $M C$ and the red pyramid is the $M C B S$. The small red circular areas are serviced by $S C B S$ which are shown as green pyramids, the solid small circles represent the $S C$ locations obtained by $k$-means algorithm, and the dashed red circles are the new locations of SCs obtained by applying the proposed algorithm. The black arrow shows the shifting in the location of a $S C$, and the black dots are the non-uniformly distributed users

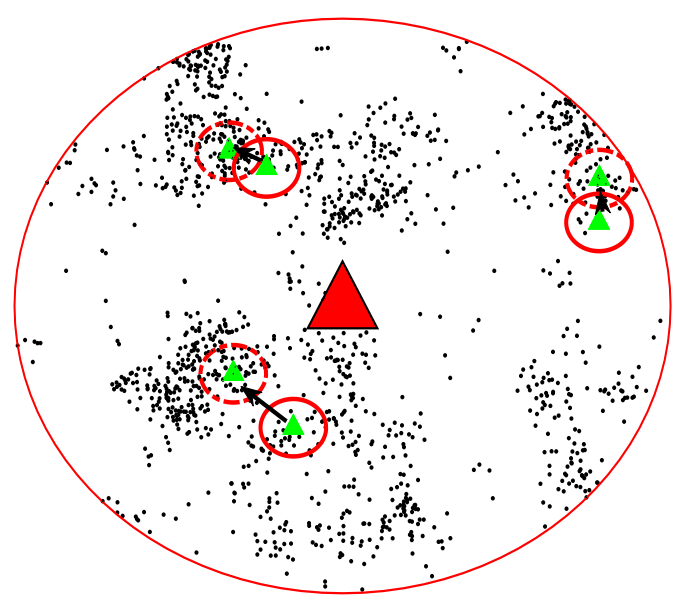

Fig. 1 An Example HetNet
The data traffic of the users under the SCs is serviced by the $S C B S s$ and the data traffic of the remaining users is serviced by the $M C B S$. The interference model assumed in this paper considers interference between $M C B S$ to different $S C B S s$, between $S C B S s$, and $S C B S s$ to $M C B S$. We propose an improved algorithm for modelling user clustering which performs better than the $k$-means algorithm. The improvement is shown by metrics that quantifies the increase in the number of $S C$ users and the increase in the data throughput of the remaining macro users. We also quantify the offloading of data traffic from the $M C B S$, and also the energy efficiency of the $S C s$ to be used as performance metrics.

It may be noted that the users are spatially clustered because of their non-uniform distribution in the coverage area. In this paper, our grouping or clustering algorithm by modelling of user spatial clustering is to find optimal locations of the SCS for deployment for efficient service coverage in the HetNet. The grouping or clustering of users, while explaining the proposed algorithm is merely to collect users in groups or clusters for downlink data transmission and reception from respective $S C s$, users' spatial geographical locations remain unaltered. Hence, the proposed algorithm may be taken to perform service clustering only. Further we considered a static case of user distribution with no mobility of users in the simulation experiment.

We state below the $k$-means clustering algorithm and the proposed improvement in the modelling of users' clustering from the $k$-means algorithm.

The basic k-means algorithm is stated as below [6]:

a. From the non-uniform user points, select $\mathrm{k}$ random points as initial centroids.

b. Repeat

i. Construct $\mathrm{k}$ clusters by assigning users closest to its centroid

ii. Recompute the centroid of each cluster.

c. Repeat until centroids do not change.

The improvement of the above algorithm is obtained as stated below.

d. Start with the centroids from the step $c$ above. 
e. Draw circles of radius 300 meters around each centroid. Size of the radius is chosen to have nooverlapping or minimal overlapping between the nearby circles around the centroids. It may be a design parameter.

f. Find which user point within 300 meters of radius of each cluster has highest number of neighbors within its 100 meters of radius.

g. The user point having highest number of neighbors around it in 100 meters of radius is taken as the new cluster centroid.

h. Repeat for every cluster from basic k-means algorithm.

The benefits of the proposed algorithm are as follows:

i. The SCs which centers are the centroids from the basic k-means algorithm may not contain any user within its 100-meter cell radius which centers are the centroids from the basic k-means algorithm may not contain any user within its 100-meter cell radius. Also, the centroids may be located in zones where users are sparsely spread.

The SC centers as obtained from the proposed algorithm contain the maximum number of user points within its 100 -meter radius. Hence, the SC centers are located in the densest sites of each cluster in proposed improved algorithm obtained from basic k-means algorithm.

ii. The offloading of traffic from the $M C B S$ is much less as the SCs obtained by k-means algorithm contain no or much less number of user points. Since, the SCs as obtained from the proposed algorithm have the maximum possible number of user points in each $S C$, the offloading of traffic from the $M C B S$ is highest possible.

iii. As offloading of data traffic from $M C B S$ is highest possible, it enhances the data throughput and data rate obtained by each macro users from its $M C B S$.

To obtain the $x$ number of $S C$ locations (centroids) in the $M C$ $B S$ coverage area, we proceed in two different methods. In the first method, we obtain $x$ number of groups or clusters of users and their centroids applying $k$-means and the proposed algorithm. We then evaluate the performance of the HetNet designed under the two algorithms. In the second method, applying the two algorithms, we start with $y$ number of $S C s$ in the $M C B S$ coverage area and then switch off some $S C s$ by $z$ numbers, where $z=1,2,3,4$ and keeping $y-z S C s$ in their original positions as per the algorithm. We then evaluate the performance of the HetNet designed under the two algorithms.

For simulation studies, we consider four different cellular HetNets with total user points in the range of 10001400, each one with one $M C B S$ and 4-8 SC BSs. The SCs are distanced from the $M C B S$ by 400 meters so that interference from the $M C B S$ to users under the $S C s$ are low. In Fig.2(a) and 2(b) we show such a cellular HetNet with 4 SCs and 7 SCs respectively, after applying the proposed algorithm. In Fig.2(c) we show the network of Fig.1(b) with 3 SCs switched off randomly. In Fig.3(a), 3(b) and 3(c), show another network with same number of $S C s$

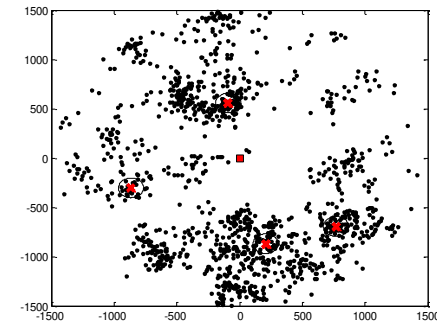

Fig.2(a) : Hetnet 1,4 SCs

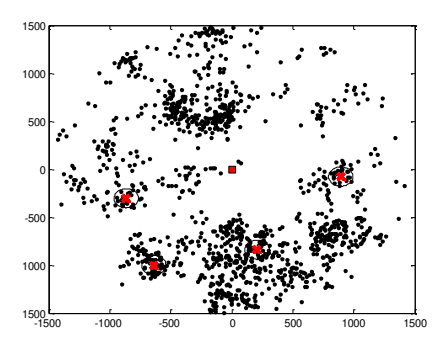

Fig.2(c) : Hetnet1, 4 SCs. 3 SCs removed randomly from a total of 7 SCs.

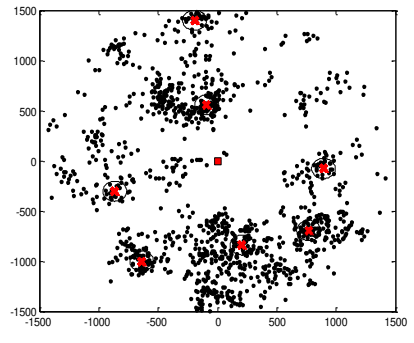

Fig.2(b) : Hetnet 1,7 SCs. 


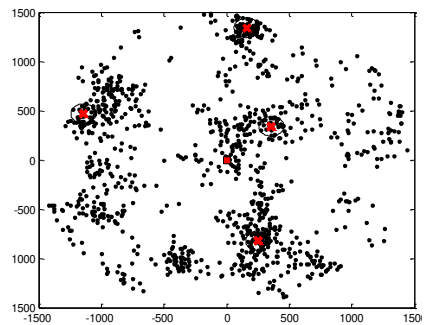

Fig3 (a) : Hetnet2, 4 SCs

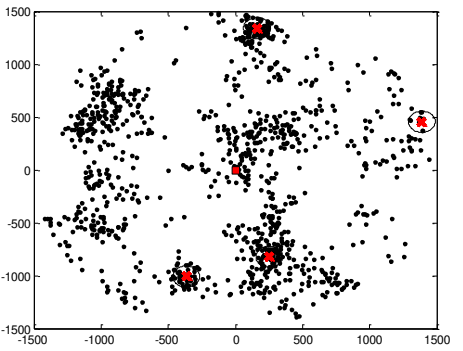

Fig.3 (c) : Hetnet2, 4 SCs. 3 SCs removed randomly from a total of 7 SCs.

The other two networks, considered for our study have been shown in the result and discussion in Fig. 4 and in Fig. 5. For throughput and data rate calculations, we consider Rayleigh distributed small scale fading model, large scale fading factor representing shadow fading, and user distances are considered as user points are at different distances from their servicing $B S s$.

\section{THEORETICAL FORMULATION OF METRICS}

In this Section, we develope four metrics, PI_MUDR, PI_SC, OFFLOAD, $P G$, and BS_SC_W for evaluating the performance of the proposed modelling of user clustering.

The downlink singal to interference and noise ratio $(S I N R)$ for the $j^{\text {th }}$ user under the service of the $M C B S$ (only one $M C B S$ ), and the $k^{\text {th }}$ user under the service of the $\mathrm{i}^{\text {th }} S C B S$ is as follows.

A. Downlink SINR for the $\mathrm{j}^{\text {th }}$ macro user, interferences from all $S C s$ :

$$
\operatorname{SINR}_{j}=\frac{\left(P_{j}\left|h_{j}\right|^{2} g_{p l, j}\right)}{\left(\sum_{i=1}^{N_{s}} P_{s}^{j}\left|h_{s, j}^{i}\right|^{2} g_{p l, j}^{i}+N_{0}\right)}
$$

The numerator in Eq. 1 stands for $j^{\text {th }} M C$ user signal from its serving $M C$, the first term in the denominator stands for the total interference from all $N_{S} S C s$ to the $j^{\text {th }} \mathrm{MC}$ user. $P_{j}$ is received power, $h_{j}$ is the small scale fading gain, and $g_{p l, j}$ is the path loss at distance $d$ from the $M C B S . \quad P_{s}{ }^{i}$ is the power received, $h_{s, j}^{i}$ is small scale fading gain, and $g_{p l, j}^{i}$ is the path loss from the $i^{\text {th }} S C B S$ to the $j^{\text {th }}$ macro user. $N_{0}$ stands for Gaussian noise power in $W$ Hertz, $N_{S}$ is total number of SCs. The path loss model is defined as:

$$
g_{p l, j}=c d_{j}^{-\alpha} \text { and } g_{p l, j}^{i}=c d_{i, j}^{-\alpha}
$$

Where, $\alpha$ is the path loss exponent, $c$ represents large scale fading factor representing shadow fading, $d_{j}$ and $d_{i, j}$ are the distances of the $j^{\text {th }}$ user from the $M C B S$ and the $i^{\text {th }} S C$ respectively.

The data rate of the $j^{\text {th }} M C$ user with bandwidth $W$ Hertz is given by

$$
\gamma_{j}=W \log _{2}\left(1+\operatorname{SINR}_{j}\right)
$$

The sum data rate of a total $n_{m}$ users under the $M C B S$ is given by:

$$
S R_{m}=\sum_{j=1}^{n_{m}} \gamma_{j}
$$

B. Downlink SINR for the $k^{\text {th }}$ user in the $i^{\text {th }} \mathrm{SC}$, interference from $M C B S$ and other SCs :

$$
\operatorname{SINR}_{k}^{i}=\frac{\left(P_{s, k}^{i}\left|h_{s, k}^{i}\right|^{2} g_{p l, k}\right)}{\left(P_{k}\left|h_{k}^{i}\right|^{2} g_{p l, k}^{i}+\sum_{l=1}^{N_{s}-1} P_{s, k}^{l}\left|h_{s, k}^{l}\right|^{2} g_{p l, k}^{l}+N_{0}\right)}
$$

The numerator of Eq. (5) stands for user received signal from its serving $S C$. In the denominator, the first term stands for the interference signal from the $M C B S$, and the second term stands for the interference signals from the rest $N s-1$ SCs. $P_{k}$ is received power, and $h_{k}{ }^{i}$ is the small scale fading gain and $g_{p l, k}{ }^{i}$ is the path loss, of the $k^{\text {th }}$ user in the $i^{\text {th }} S C$ with respect to the MC BS. $P_{s, k}{ }^{i}$ is the power received, $h_{s, k}{ }^{i}$ is small scale fading gain, and $g_{p l, k}$ is the path loss, of the $k^{\text {th }}$ user in the $i^{\text {th }} S C$. And $P_{s, k}{ }^{l}$ is the received power, $h_{s, k}{ }^{l}$ is small scale fading gain, and $g_{p l, k} l$ is the path loss, of the $k^{\text {th }}$ user in the $i^{\text {th }} S C$ with respect to the $l^{\text {th }} \mathrm{SC}$. $\left(N_{s}-1\right)$ is the number of interfering SCs, $g_{p l, k}, g_{p l, k}{ }^{i}$ and $g_{p l, k}{ }^{l}$ follow the definition in Eq. 2 
The data rate of the $k^{\text {th }}$ user in the $i^{\text {th }} \mathrm{SC}$ with bandwidth $W$ Hertz is given by:

$$
\gamma_{k}^{i}=W \log _{2}\left(1+\operatorname{SINR}_{k}^{i}\right)
$$

The sum data rate of $n_{i}$ users under the $i^{\text {th }} S C s$ is given by:

$$
S R_{s}^{i}=\sum_{k=1}^{n_{i}} \gamma_{k}^{i}
$$

The sum data rate of all users under total $N_{s}$ number of $S C s$ is given by:

$$
S R_{s}=\sum_{i=1}^{N_{s}} S R_{s}^{i}
$$

We can define the percentage increase of total $S C$ users under all SCs, PI_SCU as defined below. Let, $n_{m}^{!}, n_{m}{ }^{*}$ be the total number of macro users when clusters are constructed by kmeans clustering algorithm and the proposed algorithm, respectively. Let, $\sum_{i=1}^{N_{s}} n_{s}$ ' and $\sum_{i=1}^{N_{s}} n_{s}^{*}$ be the total number of $S C$ users under a total $N_{s}$ number of $S C s$, following basic (kmeans) and proposed clustering algorithms.

$$
P I_{-} S C U=\left(\frac{\sum_{l=1}^{N_{s}} n_{s}^{*}-\sum_{l=1}^{N_{s}} n_{s}^{!}}{\sum_{l=1}^{N_{s}} n_{s}^{!}}\right) \times 100
$$

Similarly, the percentage increase of data rates of the macro users, $P I \_M U D R$ due to the proposed clustering algorithm is defined as:

$$
P I_{-} M U D R=\left[\frac{\left(\sum_{j=1}^{n_{m}^{*}} \gamma_{j} / n_{m}^{*}\right)-\left(\sum_{j=1}^{n_{m}^{\prime}} \gamma_{j} / n_{m}^{!}\right)}{\left(\sum_{j=1}^{n_{m}^{\prime}} \gamma_{j} / n_{m}^{!}\right)}\right] \times 100
$$

Both the parameters $P I \_M U D R$ and $P I \_S C U$ are used in this paper to evaluate the performance of the proposed clustering algorithm.

The amount of data traffic difference caused by $N_{s} S C s$ from the $M C B S$ is defined as:

$$
O F L D=\left(S M M_{-} D R_{-} B\right)-\left(S M_{-} D R_{-} A\right)
$$

where, $O F L D$ is the data traffic difference, $S M \_D R \_B$ is sum data rate of total macro users before SCs are deployed, and $S M \_D R \_A$ is sum data rate of total macro users after $S C S$ are added. The total number of users under $N_{s} S C s$ in the new proposed and basic (k-means) algorithms contribute to OFLD, and are multiplied with corresponding $O F L D$ to define a new metric called OFFLOAD. Thus, OFFLOAD in the proposed algorithm and in basic (k-means) algorithm is defined as below. The parameter OFFLOAD is used as a metric in this paper to evaluate the performance of the proposed algorithm

$$
\begin{aligned}
& O F F L O A D_{N E W}=\left(S R_{n_{m}}-S R_{n_{m}^{*}}\right) \times \sum_{i=1}^{N_{s}} n_{s}^{*} \\
& O F F L O A D_{O L D}=\left(S R_{n_{m}}-S R_{n_{m}^{\prime}}\right) \times \sum_{i=1}^{N_{s}} n_{s}^{!}
\end{aligned}
$$

We also define the gain in the system data rate because of adding SCs. As is intuitively understood, there would be increase in the sum data rate of the system when service is provided by the $M C$ and the SCs together compared to the sum data rate when service is provided by the $M C$ only. The percentage gain $P G$ in the proposed algorithm, and in the basic (k-means) algorithm are defined as below:

$$
\begin{aligned}
P G_{\text {New }} & =\frac{S y s_{-} T h p_{\mathrm{New}}-T h p_{-} m c}{T h p_{-} m c} \times 100 \\
P G_{\text {Old }} & =\frac{S y s_{-} T h p_{\text {Old }}-T h p_{-} m c}{T h p_{-} m c} \times 100
\end{aligned}
$$

where, Sys_Thp $p_{\text {New }}$ and Sys_Thpold are the total throughput or sum data rate of the system with macro and SCs as per new proposed algorithm and basic (k-means) algorithm. Thp_mc is the sum data rate of the system due to $M C$ only and before addition of $S C s$.

Observation: Although we defined percentage gain, $P G$ in Eq.12, it cannot be used as a metric for evaluating the system performance. This is because the combined data throughput of all SCs in the basic (k-means) clustering algorithm may happen to be several times higher, compared to the case in the improved proposed algorithm, as the number of users under a $S C$ may be very small. The greatest disadvantage of the kmeans clustering algorithm is that the centroid of the cluster may be quite separated from its members. Also, the $S C$ which is placed at the centroid has small coverage area. The observed 
effect on the data throughput of all users under a $S C$ and hence of all $S C s$ is the outcome of round robin user scheduling assumed here for data transmission by a $B S$. Hence, as shown in Eq. 12, $P G_{N e w}$ is expected to be smaller than $P G_{\text {Old }}$, as Sys_Thp $p_{\text {New }}<S y s \_T h p_{\text {Old }}$. This is further explained in the next section.

As more users are included under a $S C$ in the proposed clustering algorithm compared to the basic (k-means), we define energy efficiency for SCs considering together as below:

$$
\begin{aligned}
& B S_{-} S C_{-} W_{\text {New }}=S C_{-} S T H P_{\text {New }} / T P S \\
& B S_{-} S C_{-} W_{\text {Old }}=S C_{-} S T H P_{\text {Old }} / T P S
\end{aligned}
$$

Where, $B S \_S C \_W_{\text {New }}$ and $B S \_S C \_W_{\text {old }}$ are the sum data rate of the SCs per watt as per the proposed and basic $(\mathrm{k}$ means) clustering algorithms, respectively. $S C_{-} S T H P_{N e w}$ and $S C_{-} S T H P_{\text {Old }}$ are the sum data rate of the SCs together as per the proposed and basic (k-means) clustering algorithm, respectively, TPS is the total transmission power of the whole $S C B S$ s together. The performance of the proposed clustering algorithm is evaluated using energy efficiency for SCs as defined in Eq.13.

\section{SimUlation ResUlts AND DisCUSSIONS}

We consider that the $M C B S$ and all $S C B S s$ service data traffic to their users independent of each other, and the users under a $B S$ are scheduled using round robin algorithm. As the users under the macro cell are large in numbers, the round robin time frame length is much longer and results in smaller individual user data throughputs. This is opposite in SCs where the round robin time frame length is much smaller resulting in much higher individual user data throughputs. Further, for simulation studies, we consider the positions of the users and hence their distributions shown in various networks remain static. We consider Rayleigh distributed small scale fading model, large scale fading factor representing shadow fading, and users' geographical distances from $B S s$ to evaluate users' data rates
We consider 4 different HetNets, each in a 1500-meter circular geographical area and each with $1 M C B S$ and 4-8 SC $B S s$. The $M C B S$ is located at center and SC BSs are located at the centroids of the clusters. We first apply the k-means algorithm, thereby obtaining the locations for the $S C B S s$. We then apply the proposed algorithm to the cluster points from $k$ means algorithm, thereby obtaining the new locations of the $S C B S s$

Table 1

\begin{tabular}{|l|l|}
\hline \multicolumn{1}{|c|}{ Parameters } & \multicolumn{1}{c|}{ Values } \\
\hline Path loss exponent $(\alpha)$ & 2.5 \\
\hline Large scale fading factor $(c)$ & $10^{-3.53}$ \\
\hline Bandwidth $(W)$ & $180 \mathrm{KHz}$ \\
\hline Time slot for service & $0.5 \times 10^{-3}$ seconds \\
\hline Noise power spectral density & $-174 \mathrm{dBm} / \mathrm{Hz}$ \\
\hline Transmit power of MC BS & $46 \mathrm{dBm}$ \\
\hline Transmit power of SC BSs & $23 \mathrm{dBm}$ \\
\hline Radius of MC & $1500 \mathrm{~m}$ \\
\hline Radius of a SC & $100 \mathrm{~m}$ \\
\hline
\end{tabular}

In each case, the users within an area of 100 meter of the locations of the $S C B S s$ are taken to be the users for service by the SCs. The remaining users in the circular geographical area are serviced by $M C B S$. Parameters for the HetNets considered in this paper are shown in Table 1.

Of the 4 different HetNets, two are shown in Fig.2, and Fig.3 in the section of System Model above. The other two networks are shown in Fig.4, and Fig.5 below. Fig. 4 (c) and Fig. 5 (c) shows remaining 4 SCs after 3 SCs are switched off randomly from Fig. 4 (b) and Fig. 5 (b), respectively.

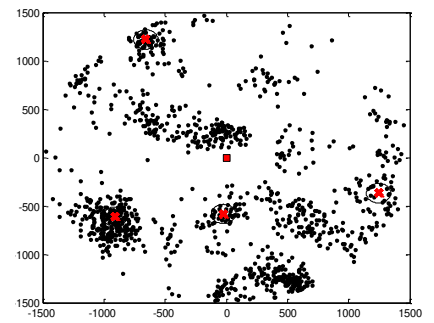

Fig.4 (a): HetNet 3, 4 SCs

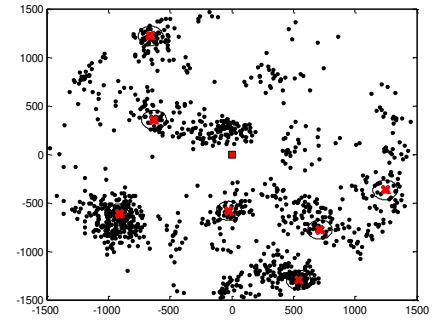

Fig.4 (b): HetNet 3, 7 SCs 


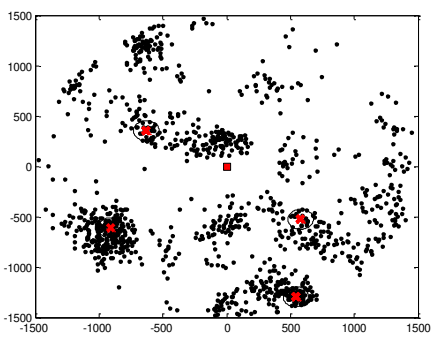

Fig.4 (c): HetNet 3, 4 SCs. 3 SCs removed randomly from a total of 7 SCs.

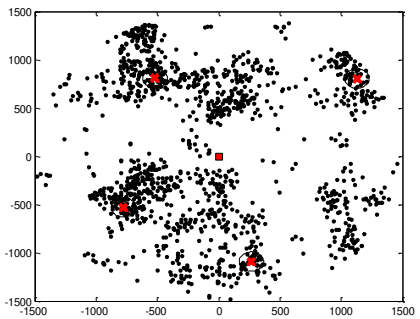

Fig.5 (a): HetNet 4, 4 SCs

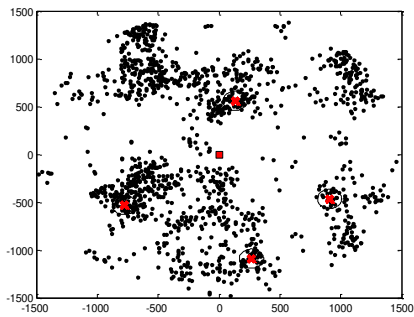

Fig.5 (c): HetNet 4, 4 SCs. 3 SCs removed randomly from a total of 7 SCs

The experimental simulation results of PI_MUDR and PI_SCU for the four HetNets are shown in Fig.6 and Fig.7, using Eq. 8 and Eq. 9 respectively. It is observed that there are considerable increase in the $M C$ user data rates, and in the number of users in different SCs. The increasing trend is shown in case of all HetNets.

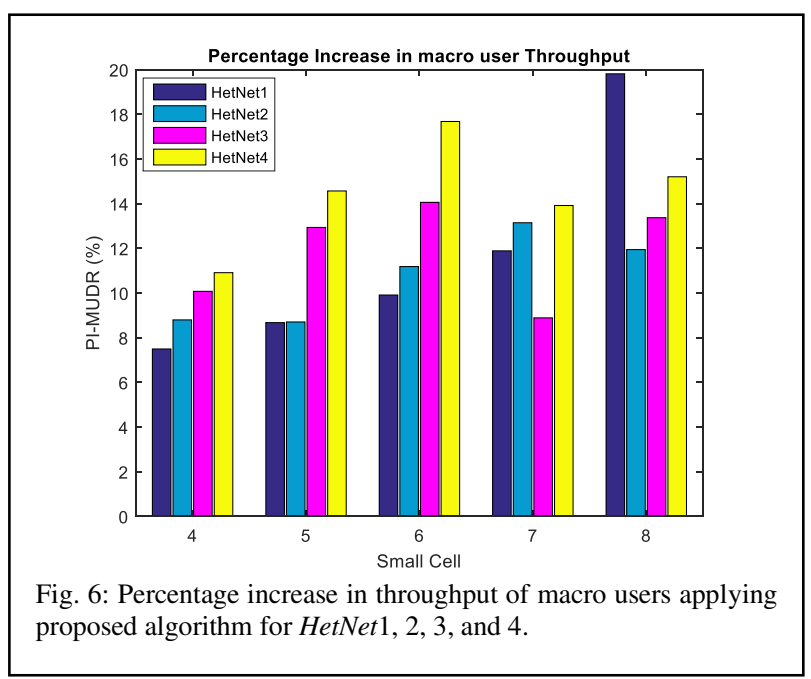

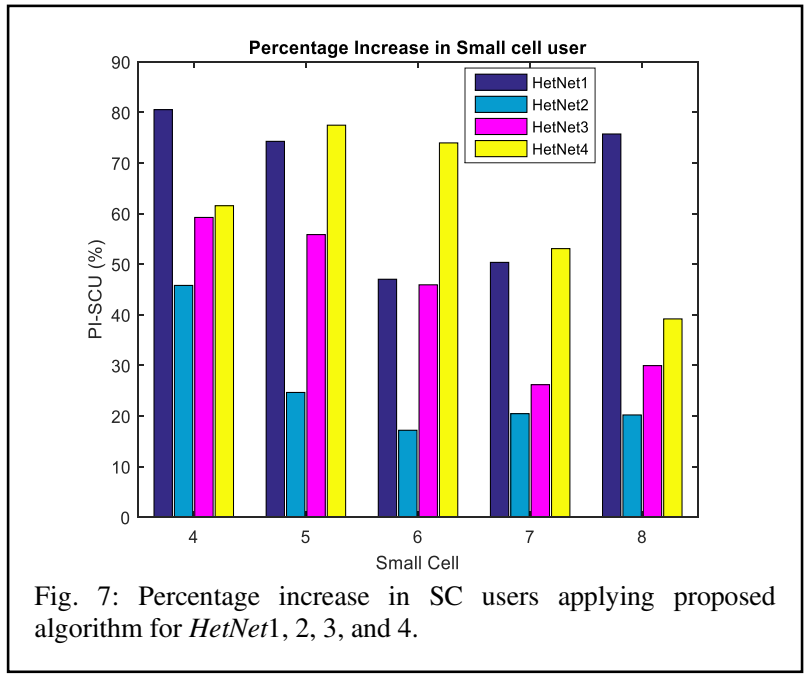

The results of using the proposed algorithm in all 4 HetNets are shown below by simulation in the MATLAB. To evaluate the proposed algorithm, the parameter OFFLOAD, using the proposed and basic (k-means) clustering algorithms as in Eq.11, experimental simulation results for the four HetNets are shown in Fig. 8 (i-iv) as bar diagrams. The metric shows clear advantage in using the proposed algorithm. The increasing trend is shown in case of all HetNets.

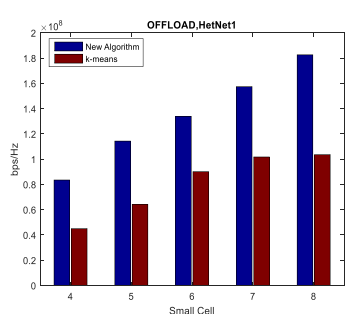

Fig. 8 (i): Data offloaded by $S C s$ in HetNet 1 applying proposed algorithm and k-means algorithm

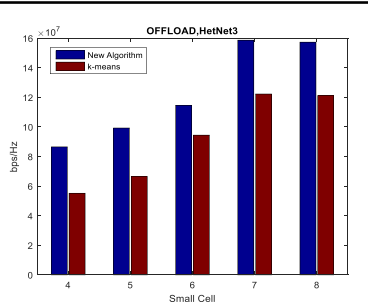

Fig. 8 (iii): Data offloaded by $S C s$ in HetNet3 applying proposed algorithm and k-means algorithm

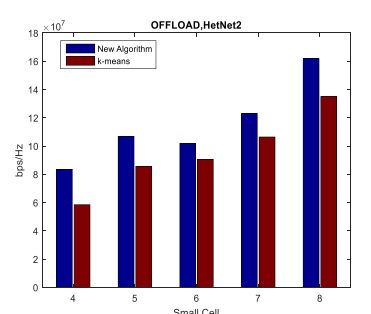

Fig. 8 (ii): Data offloaded by $S C s$ in HetNet2 applying proposed algorithm and k-means algorithm

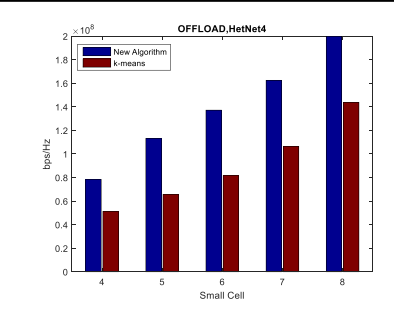

Fig.8 (iv): Data offloaded by $S C s$ in HetNet4 applying proposed algorithm and k-means algorithm 
As is observed in the discussion of Eq.12, the percentage gain, $P G$ in the sum data rate of the system when service is provided by the $M C$ and the $S C s$ together compared to the sum data rate when service is provided by the $M C$ only is worse while using the proposed algorithm. The reason is explained in the observation. The experimental simulation results for the four HetNets are shown in Fig. 9 (i-iv).
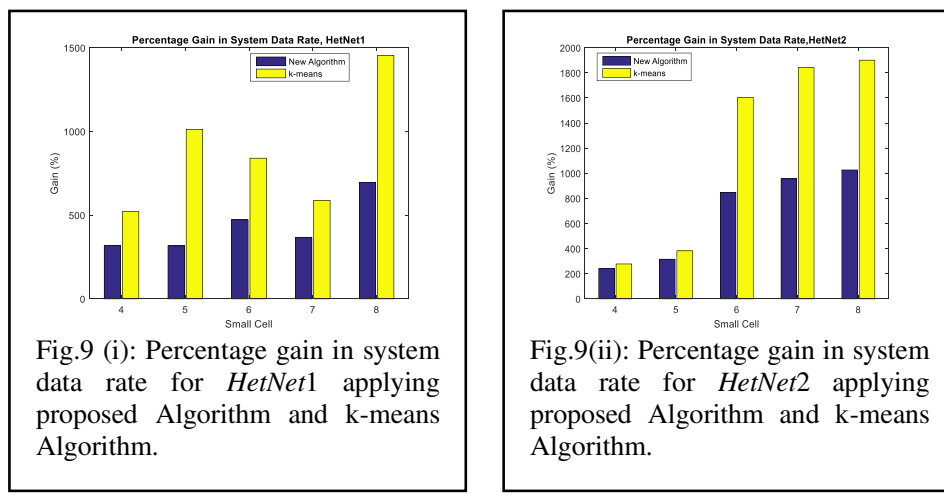

Fig.9(ii): Percentage gain in system data rate for HetNet2 applying proposed Algorithm and k-means Algorithm.

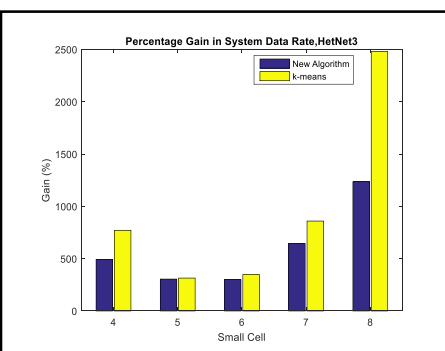

Fig.9 (iii): Percentage gain in system data rate for HetNet3 applying proposed Algorithm and k-means Algorithm

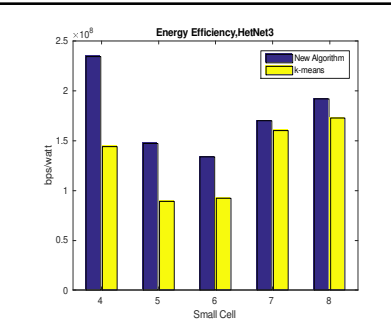

Fig.10 (iii): Energy Efficiency algorithm and k-means algorithm in HetNet3 applying proposed

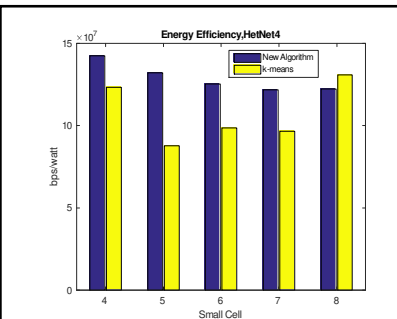

Fig. 10 (iv): Energy Efficiency in HetNet4 applying proposed algorithm and k-means algorithm
As stated in Section 2, for the second method of HetNet design, following the $k$-means and the proposed algorithm, we start with $y$ number of SCs where $y=8$ and switch off $z$ number of SCs where $z=1,2,3,4$ in steps. The remaining SCs $y-z=x$, where $x=7,6,5,4$ in the HetNet are the results of randomly switching off $S C s$ from the initial scenario of total 8 SCs. The simulation results of switching off $z$ number of SCs are shown in Table 2 and Table 3. All four metrics are dependent on SCs that are switched off from initial 8 SCs. We are of the opinion that the decision of which SCS may be switched off depends on certain factors which may be technical, financial as well as administrative. Here we switched off SCs randomly with no obvious reason.

The simulation results of PI_MUDR, PI_SCU, as shown in
Fig.9 (iv): Percentage gain in system data rate for HetNet4 applying proposed Algorithm and k-means Algorithm
The performance metrics $B S \_S C_{-} W_{\text {Old }}$ and $B S \_S C_{-} W_{\text {New }}$, the sum data rate of the SCs per watt using the basic (k-means) and new proposed algorithm, respectively are evaluated for the four HetNets. The experimental simulation results are shown in Fig. 10 (i-iv) using Eq.13. As is observed from the bar diagrams, there is increase in the energy efficiency of the combined SCs in all the four HetNets.
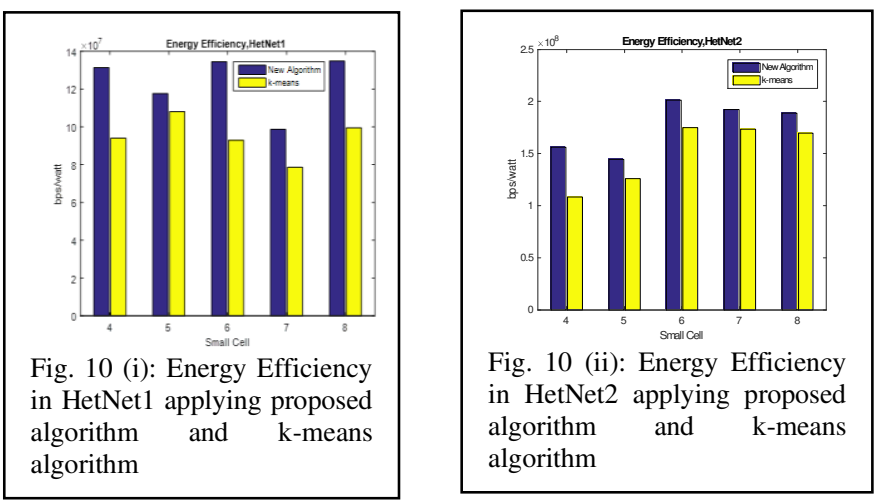

Table 2 are expected to be different from the results shown in Fig. 6 and Fig. 7. Similarly, the simulation results of $B S \_S C \_W_{N e w}$, and $B S \_S C \_W_{\text {old }}$ as shown in Table 3 are expected to be different from the results shown in Fig. 10. Hence, the values of all four metrics are also dependent on the user distributions in the HetNets. However, the proposed algorithm always provides positive values of the metrics studied in this paper. As there are a greater number of users in $S C s$, the centers of which are obtained by using the proposed algorithm, the data traffic offloading from the macro $B S$ by the $S C s$ is always larger than the case of using k-means algorithm. The way how we retain or design HetNet for a particular number of $S C B S s / S C s$ may decide the performance of HetNets. The design objectives should satisfy the required 
service coverage or total transmit power available for the $S C$

BSs.

TABLE-2

\begin{tabular}{|l|l|l|l|l|l|l|l|l|}
\hline $\begin{array}{c}\text { No. } \\
\text { of } \\
\text { SCs. }\end{array}$ & \multicolumn{2}{|c|}{$\begin{array}{c}\text { HetNet 1 } \\
\text { PI_MUDR }\end{array}$} & \multicolumn{2}{c|}{$\begin{array}{c}\text { HetNet 2 } \\
\text { PI_MUDR }\end{array}$} & \multicolumn{2}{c|}{$\begin{array}{c}\text { HetNet 3 } \\
\text { PI_MUDR }\end{array}$} & \multicolumn{2}{c|}{$\begin{array}{c}\text { HetNet 4 } \\
\text { PI_MUDR }\end{array}$} \\
\hline 8 & 19.85 & 75.71 & 12.16 & 20.2 & 13.07 & 29.95 & 14.43 & 39.18 \\
\hline 7 & 18.75 & 70.59 & 9.56 & 16.41 & 9.15 & 22.95 & 13.83 & 37.58 \\
\hline 6 & 15.14 & 82.08 & 7.17 & 13.19 & 8.31 & 20.79 & 8.94 & 39.23 \\
\hline 5 & 9.49 & 70.1 & 4.84 & 14.84 & 3.37 & 6.85 & 6.72 & 35 \\
\hline 4 & 7.21 & 60.49 & 2.77 & 12.93 & 1.13 & 3.48 & 5.31 & 34.34 \\
\hline
\end{tabular}

TABLE-3: Energy Efficiency (EE)

\begin{tabular}{|c|c|c|c|c|c|c|c|c|}
\hline \multirow{2}{*}{$\begin{array}{c}\text { Number } \\
\text { of SCs. }\end{array}$} & \multicolumn{2}{|c|}{ HetNet1 } & \multicolumn{2}{c|}{ HetNet2 } & \multicolumn{2}{c|}{ HetNet3 } & \multicolumn{2}{c|}{ HetNet4 } \\
\cline { 2 - 9 } & $\begin{array}{c}\text { Proposed } \\
\text { Algorithm }\end{array}$ & $\begin{array}{c}k \text {-means } \\
\text { Algorithm }\end{array}$ & $\begin{array}{c}\text { Proposed } \\
\text { Algorithm }\end{array}$ & $\begin{array}{c}k \text {-means } \\
\text { Algorithm }\end{array}$ & $\begin{array}{c}\text { Proposed } \\
\text { Algorithm }\end{array}$ & $\begin{array}{c}k \text {-means } \\
\text { Algorithm }\end{array}$ & $\begin{array}{c}\text { Proposed } \\
\text { Algorithm }\end{array}$ & $\begin{array}{c}k \text {-means } \\
\text { Algorithm }\end{array}$ \\
\hline 4 & $1.2 \times 10^{8}$ & $1.1 \times 10^{8}$ & $2.09 \times 10^{8}$ & $1.77 \times 10^{8}$ & $1.28 \times 10^{8}$ & $1.27 \times 10^{8}$ & $1.09 \times 10^{8}$ & $1.42 \times 10^{8}$ \\
\hline 5 & $1.32 \times 10^{8}$ & $0.94 \times 10^{8}$ & $1.97 \times 10^{8}$ & $1.74 \times 10^{8}$ & $1.49 \times 10^{8}$ & $1.48 \times 10^{8}$ & $1.17 \times 10^{8}$ & $1.33 \times 10^{8}$ \\
\hline 6 & $1.24 \times 10^{8}$ & $0.93 \times 10^{8}$ & $1.89 \times 10^{8}$ & $1.76 \times 10^{8}$ & $1.62 \times 10^{8}$ & $1.61 \times 10^{8}$ & $1.26 \times 10^{8}$ & $1.42 \times 10^{8}$ \\
\hline 7 & $1.31 \times 10^{8}$ & $0.94 \times 10^{8}$ & $1.85 \times 10^{8}$ & $1.68 \times 10^{8}$ & $1.76 \times 10^{8}$ & $1.71 \times 10^{8}$ & $1.28 \times 10^{8}$ & $1.37 \times 10^{8}$ \\
\hline 8 & $1.35 \times 10^{8}$ & $0.99 \times 10^{8}$ & $1.89 \times 10^{8}$ & $1.69 \times 10^{8}$ & $1.92 \times 10^{8}$ & $1.92 \times 10^{8}$ & $1.22 \times 10^{8}$ & $1.31 \times 10^{8}$ \\
\hline
\end{tabular}

\section{CONCLUSION}

We proposed an improved grouping or clustering algorithm of user points, non-uniformly distributed (Cox process model) in a circular area, over the k-means algorithm. We use the algorithm to obtain the location centers of SCs which overlays the $M C B S$ area. In the given circular service area of the $S C$, maximum possible number of user points are obtained. The improved cluster centers (centroids) are obtained from the initial spatial clusters. We first construct users' groups using the $k$-means algorithm, then an area of radius 300 meter is considered around the initial k-means centroids. As the SCs are deployed to service the hot spots, edge users, or in general to offload the data traffic from the $M C B S$, location of $S C s$ should cover maximum possible users within its service area. By doing so the offloading is maximum possible, and hence increase in the data rate per macro user happens to be maximum possible. We define in this paper two metrics like PI_MUDR, PI_SCU, where the first one quantifies the percentage increase in per macro user data rates and the second one quantifies the percentage increase in the total number of users under all SCs. We also define two more metrics OFFLOAD, and $B S \_S C_{-} W$, where the first one characterizes the advantages in deploying the $S C s$ and the second one characterizes the energy efficiencies of the SCs, resulting from application of the proposed algorithm. The simulation results are shown for 4 different HetNets with 4-8 number of SCs, with 1000-1400 user points constructed using Cox process. The results show the validity of the claim that the proposed algorithm is an improved one over the basic $k$-means clustering algorithm. We also experimented with random switching off some SCs observing that the performances of HetNets remain similar. We intend to explore this further in our future report. We also intend to consider spatial mobility of users within the $M C$ service area.

\section{DECLARATION}

I. Funding - Not Applicable 
II. Conflicts of Interest/Competing Interest - No conflicts of Interest.

III. Availability of data and material - Standard parameters are used and algorithm developed is of our own.

IV. Code availability - For simulation Matlab is used.

\section{REFERENCES}

[1] Cisco, ICisco visual networking index: Global mobile data trafic forecast update, 2013-2018," http://www.cisco.com/c/en/us/solutions/serviceprovider/ visual-networking-index-vni/white-paperlisting.html, Feb. 2014, link veri_ed on June. 30th, 2014.

[2] Hughes M. and Jovanovic V., "Small cells-effective capacity relief option for heterogeneous networks," in Proc. IEEE Veh. Technol. Conf.(VTC Fall), Sept. 2012, pp. 1-6.

[3] Claussen H, Lopez-Perez D, Ho L, Razavi R, Kucera S., "Small Cell Networks: Deployment, Management, and Optimization." Hoboken, NJ:Wiley-IEEE Press; 2017.

[4] Chu NEX, Zhang J., "Small cell deployment over existing heterogeneous networks," IET Electron Lett. 2016;52(3):241-243.

[5] ElSawy H, Hossain E, Haenggi M., "Stochastic geometry for modeling, analysis, and design of multitier and cognitive cellular wireless networks: a survey," IEEE Commun Surv Tutor. 2013;15(3):9961019

[6] Z. Wang, R. Schoenen, H. Yanikomeroglu and M. StHilaire, "The impact of user spatial heterogeneity in heterogeneous cellular networks," 2014 IEEE Globecom Workshops (GC Wkshps), Austin, TX, 2014, pp. 1278-1283.

[7] Qutqut MH, Abou-zeid H, Hassanein HS, Rashwan AM, Al-Turjman FM., "Dynamic small cell placement strategies for LTE heterogeneous networks," Proc. 2014 IEEE Symposium on Computers and Communications (ISCC), Funchal, 2014, pp. 1-6.

[8] Wentao Zhao et.al., "Approximation Algorithms for Cell Planning in Heterogeneous Networks", IEEE Trans. On Vehicular Technology, vol.66, no.2, February 2017.

[9] Welton Araujo et.al, "Deployment of Small Cells and a Transport Infrastructure Concurrently for Nextgeneration Mobile Access Networks", PLoS One.2018; 13(11): e0207330.

[10] D. Abonyi, "A Novel Strategy for Prompt Small Cell Deployment in Heterogeneous Networks,"
Advances in Science, Technology and Engineering Systems Journal, Vol.4, No.4, 265-270 (2019).

[11] 3GPP, " Small Cell Enhancements for E-UTRA and EUTRAN ; Physical Layer aspects, (Release 12)," 3GPP TR 36.872, 2013

[12] 3GPP R1-130744, "WF on Evaluation Assumptions for SCE Physical Layer," Huawei, HiSilicon, CATR, CMCC, 2013.

[13] Jacob Bien, Robert Tibshirani, "Hierarchical Clustering with Prototypes via Minimax Linkage," Journal of the American Statistical Association, Vol.106, No.495, pp.1075-1084, 2011

[14] Michal Yemini, Andrew J. Goldsmith, "Virtual Cell Clustering with Optimal Resource Allocation to Maximize Cellular System Capacity," GLOBCOM 2019, DOI: 10.1109/GLOBECOM38437.2019.9014051

[15] W. Vickrey, "Counterspeculation, auctions, and competitive sealed tenders," The Journal of Finance, vol. 16, no. 1, pp. 8-37, 1961

[16] Xiaodong Xu et.al., "Reverse Auction based Green Offloading Scheme for Small Cell Heterogeneous Networks," Mobile Information systems, Volume 2016, Article ID 5087525

[17] Muhammad Hawasli, , Sultan Aldırmaz Çolak, "Toward Green Heterogeneous Small-Cell Networks: Power Optimization Using Load Balancing Technique," International Journal of Electronics and Communications, 82, 474-485, https://doi.org/10.1016/j.aeue.2017.09.012.

[18] Andrews JG, Claussen H, Dohler M, Rangan S, Reed MC, "Femtocells: past, present, and future," IEEE J Select Areas commun.2012;30(3):497-508.

[19] Nan E, Chu X., “ Stochastic geometry analysis and additional small cell deployment for HetNets affected by hot spots," Mobile Information Systems, vol. 2016, Article ID 9727891, 9 pages, Jan. 2016.

[20] S. W. Heath, M. Kountouris, and T. Bai, "Modeling heterogeneous network interference using Poisson point processes," IEEE Trans. Signal Process, vol. 61, no. 16, pp. 4114-4126, Aug. 2013

[21] ElSawy H, Hossain E, Haenggi M., "Stochastic geometry for modeling, analysis, and design of multitier and cognitive cellular wireless networks: a survey," IEEE Commun Surv Tutor. 2013;15(3):9961019.

[22] Wang Y, Zhu Q., "Modeling and analysis of small cells based on clustered stochastic geometry," IEEE Commun Lett. 2017;21(3):576-579

[23] ElSawy, H., Sultan-Salem, A., Alouini, M., et al., "Modeling and analysis of cellular networks using 
stochastic geometry: a tutorial," IEEE Commun. Surv. Tutor., 2017, 19, (1), pp. 167-203

[24] Borah J, Hussain Md A., Bora J., “ Enhancement of throughput for cellular data network by small cell deployment," IEEE International Conference on Recent Innovations in Electrical, Electronics \& Communication Engineering (ICRIEECE); July,2018,

DOI:10.1109/ICRIEECE44171.2018.9009311

[25] Lee, C.H., Lee, S.H., Go, K.C., et al., "Mobile small cells for further enhanced $5 \mathrm{G}$ heterogeneous networks", ETRI J., 2015, 37, (5), pp. 856-866

[26] Yuan Wu et.al, "Traffic Offloading in Heterogeneous Cellular Networks," Radio Resource Management for Mobile Traffic Offloading in Heterogeneous Cellular Networks, 2017 pp 1-16. 
Figures

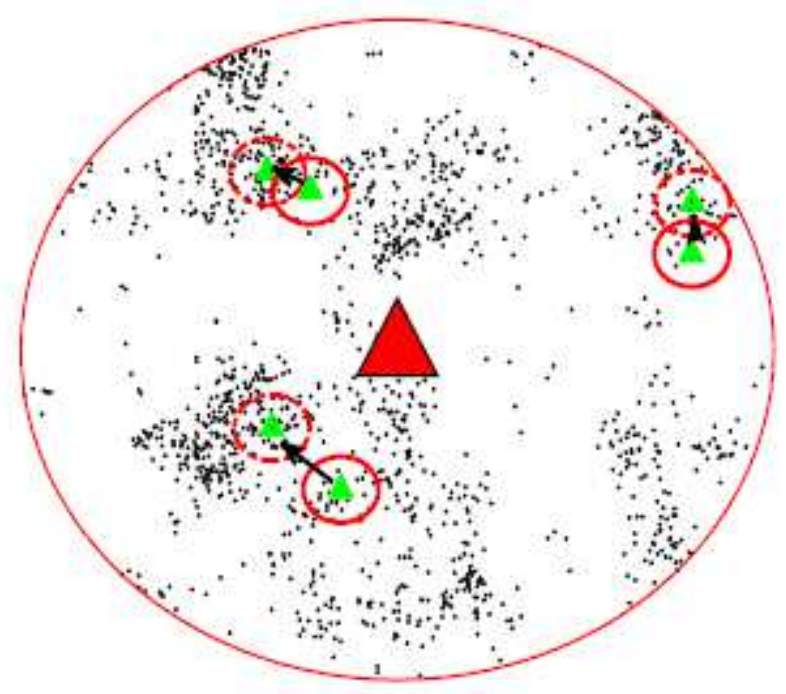

\section{Figure 1}

See the Manuscript Files section for the complete figure caption.
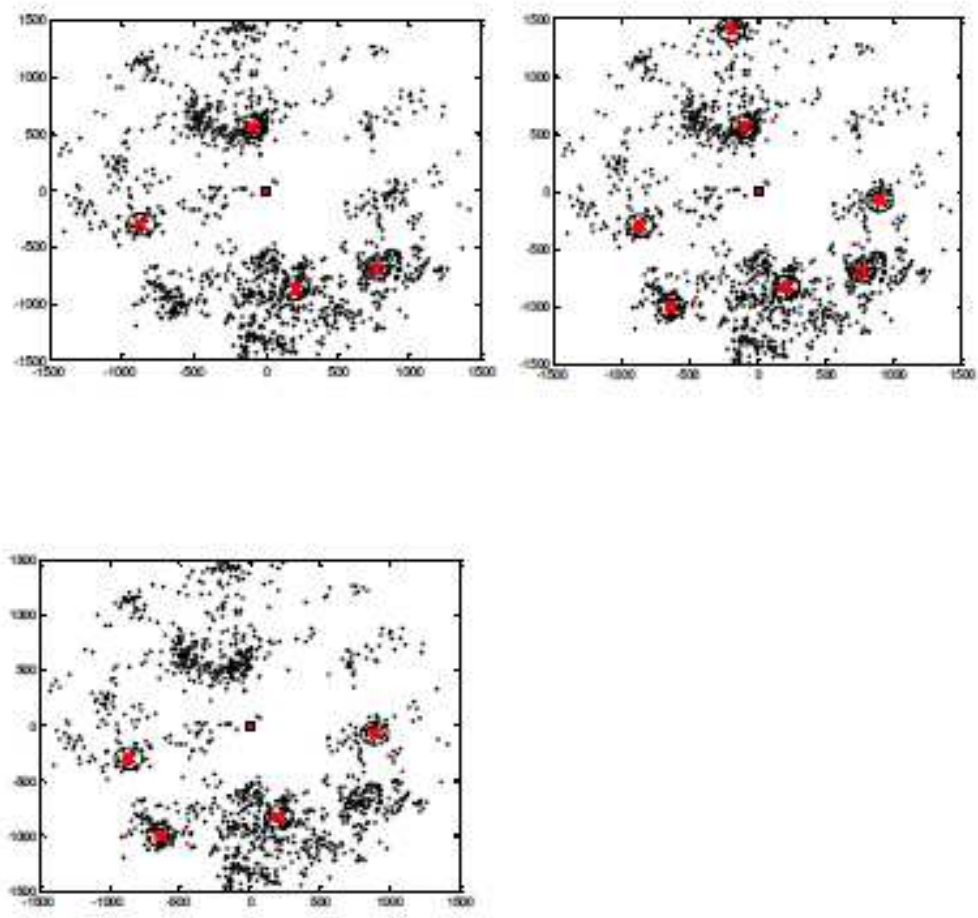

Figure 2

See the Manuscript Files section for the complete figure caption. 

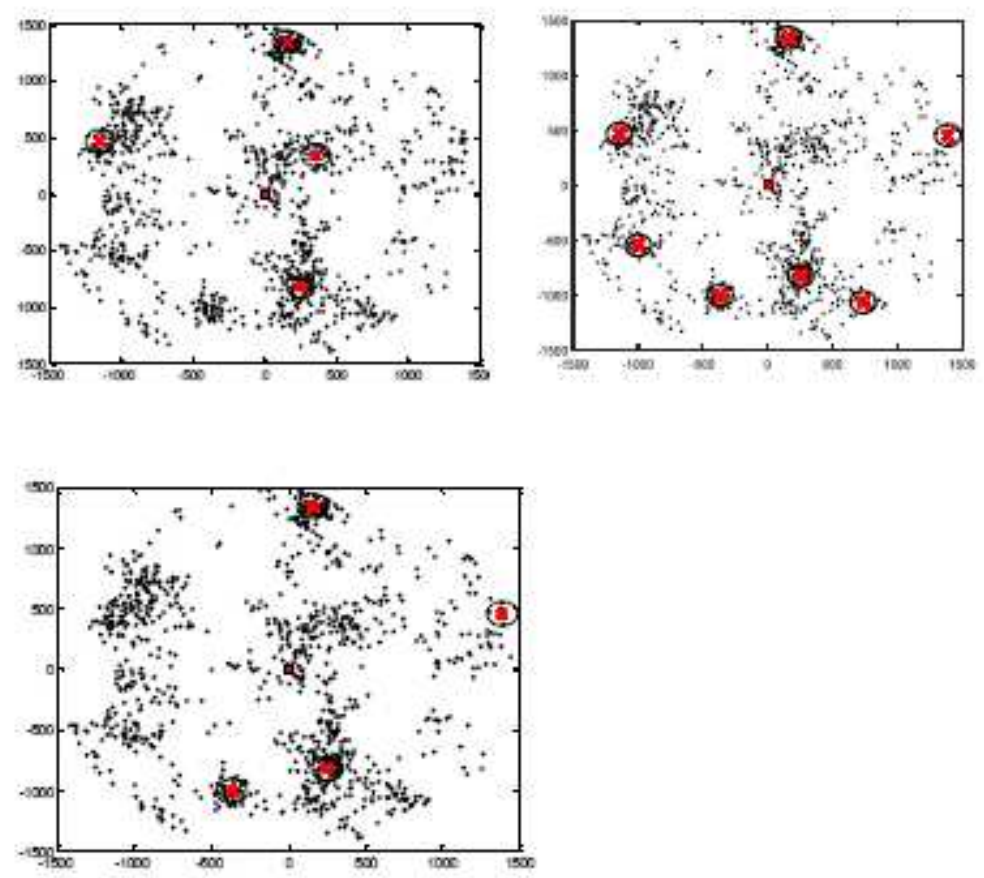

Figure 3

See the Manuscript Files section for the complete figure caption.
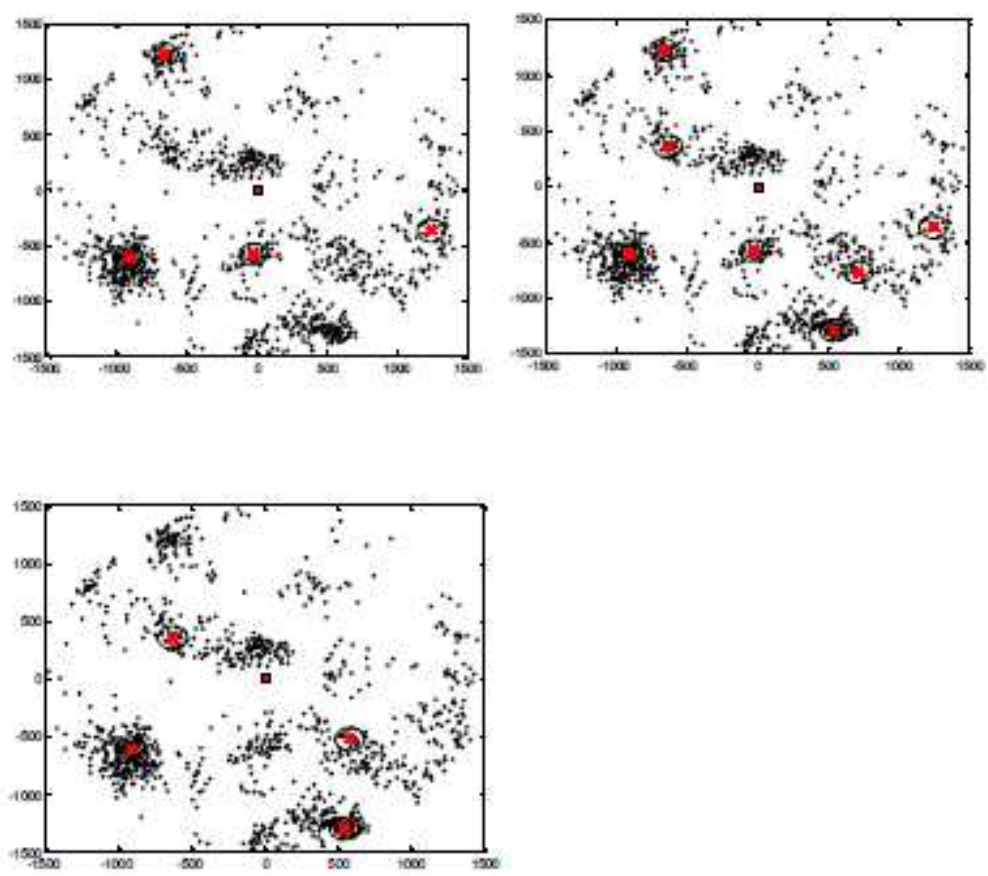

Figure 4

See the Manuscript Files section for the complete figure caption. 

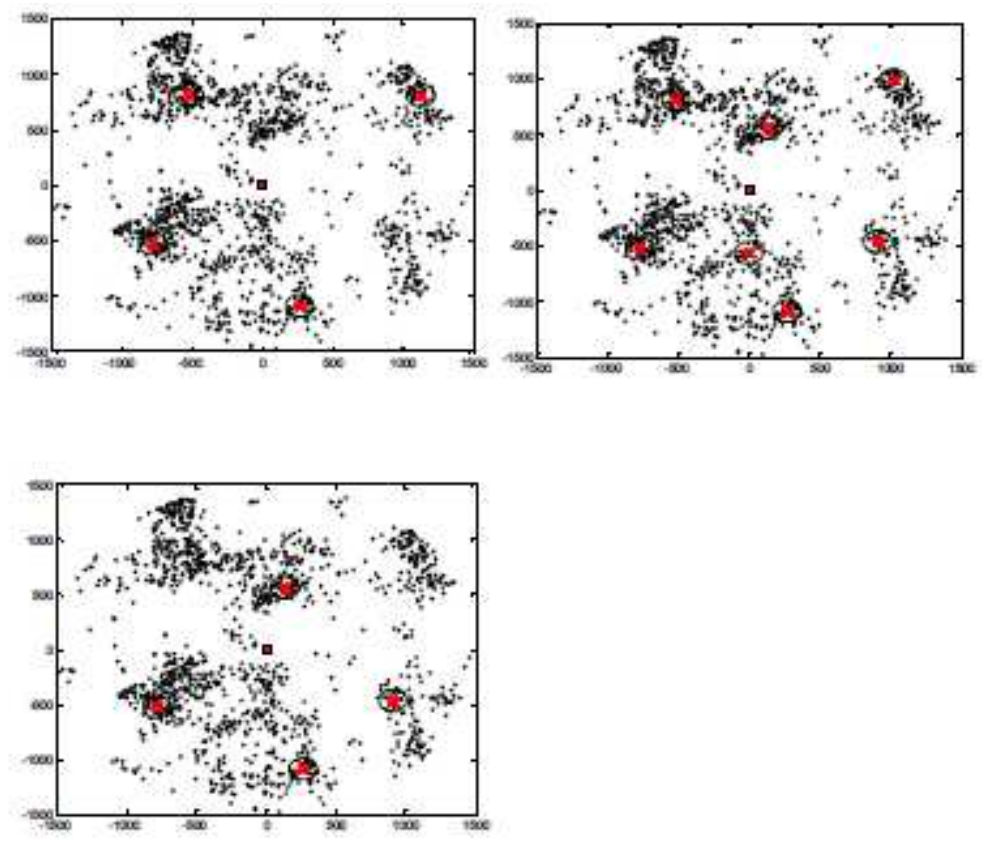

Figure 5

See the Manuscript Files section for the complete figure caption.

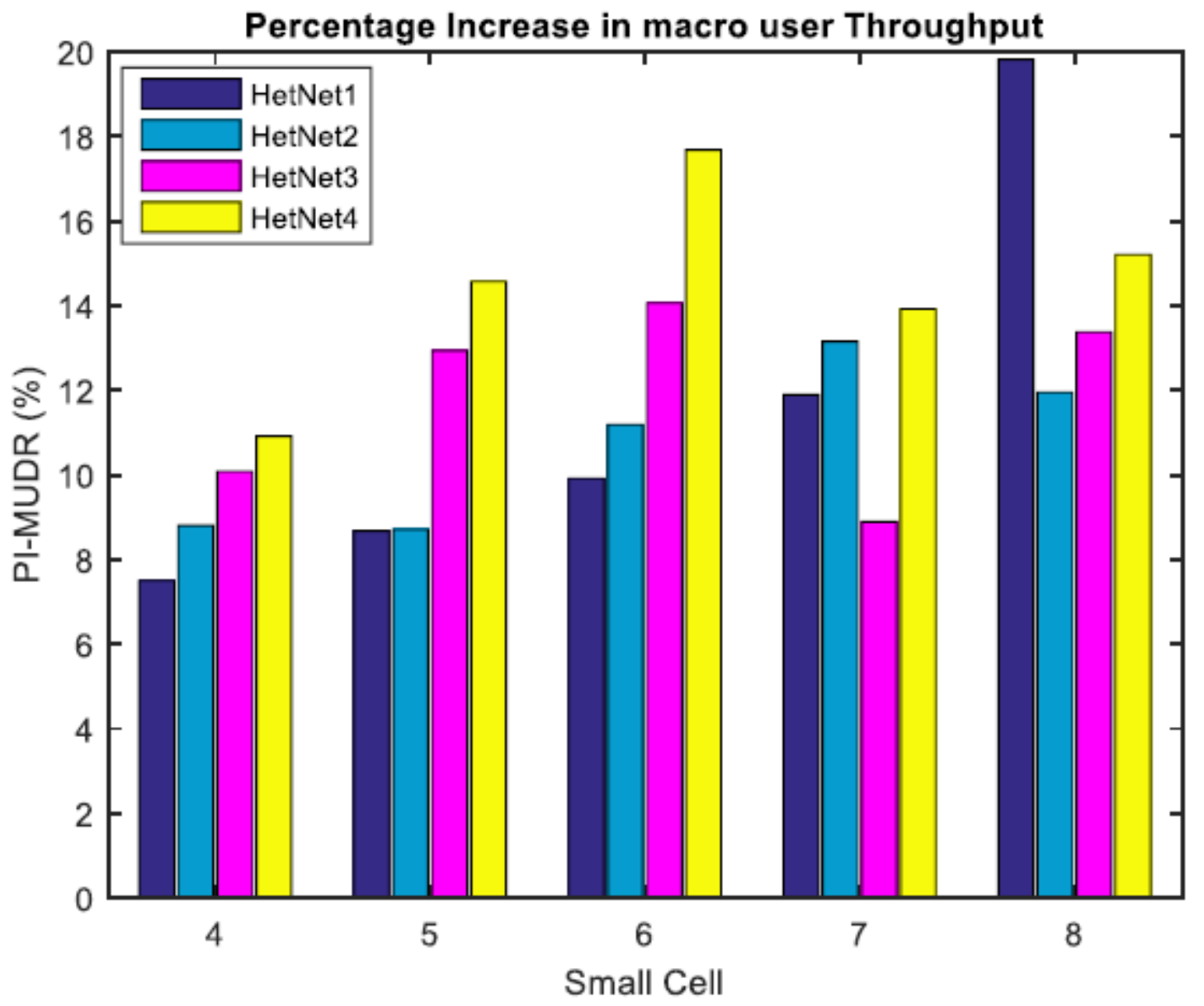

Figure 6 
See the Manuscript Files section for the complete figure caption.

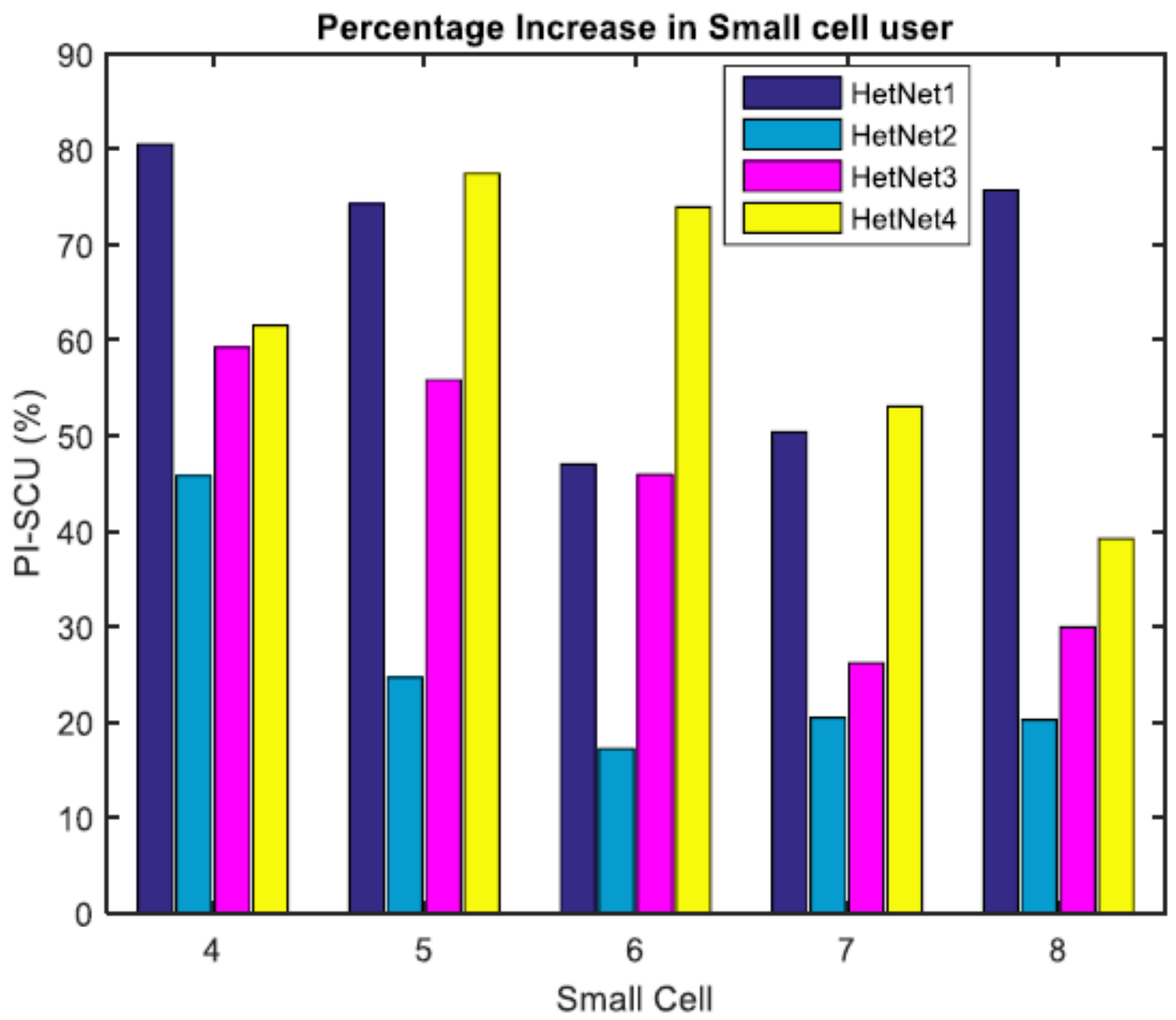

Figure 7

See the Manuscript Files section for the complete figure caption. 

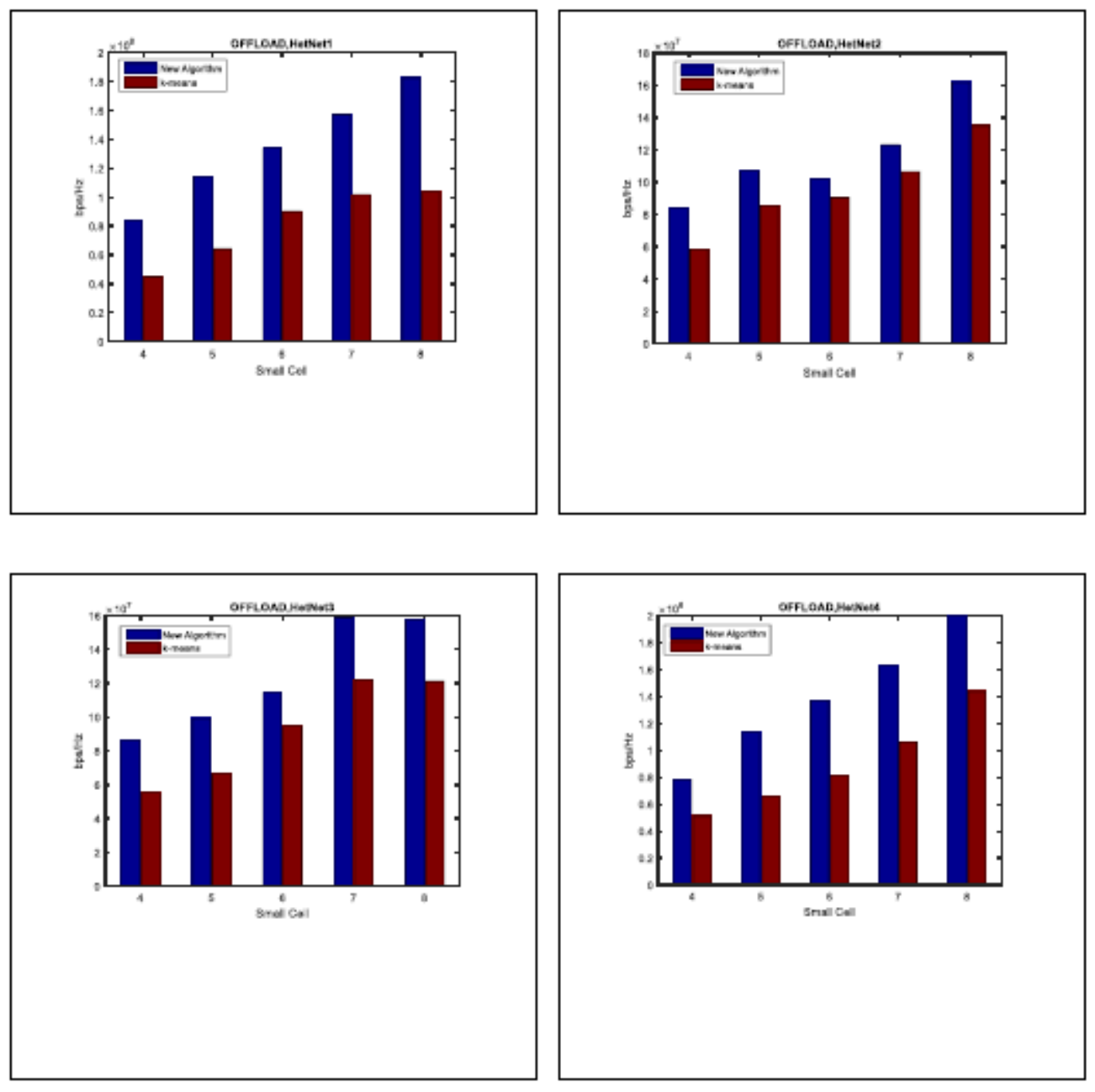

Figure 8

See the Manuscript Files section for the complete figure caption. 

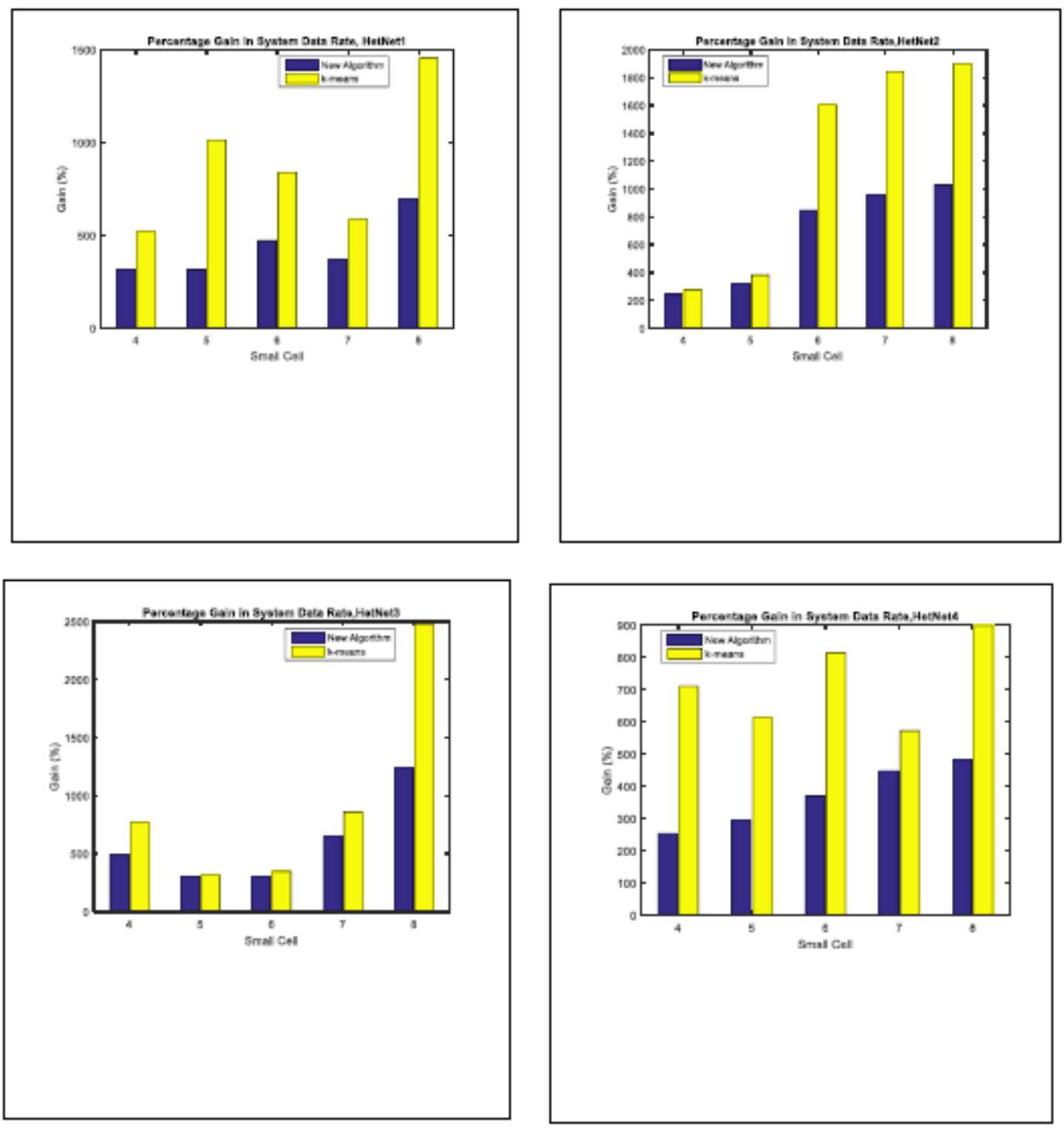

Figure 9

See the Manuscript Files section for the complete figure caption. 

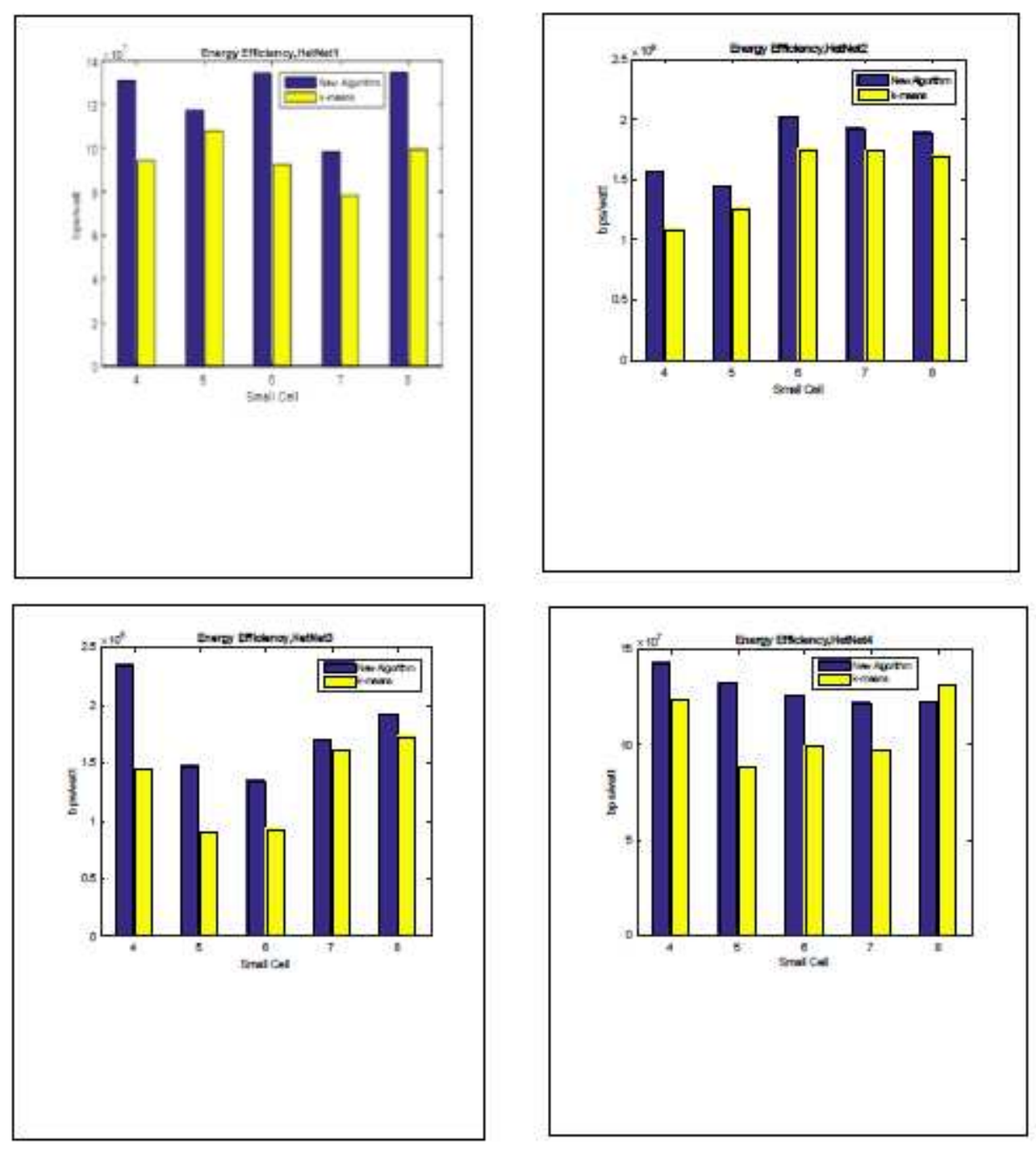

Figure 10

See the Manuscript Files section for the complete figure caption. 